\title{
C-enhanced metal poor stars and AGB nucleosynthesis at low $\mathrm{Z}$
}

\author{
Sara Lucatello $^{1}$, Raffaele Gratton ${ }^{1}$, Eugenio Carretta ${ }^{2}$, \\ and Timothy Beers ${ }^{3}$ \\ ${ }^{1}$ INAF-OAPD, vicolo dell'Osservatorio 5, 35122, Padova, Italy \\ email: lucatello@pd.astro.it \\ ${ }^{2}$ INAF-OABO, via Ranzani 1, 40127, Bologna, Italy \\ ${ }^{3}$ Michigan State University, East Lansing MI 48824, USA
}

\begin{abstract}
The study of C-enhanced metal poor stars with $s$-process elements overabundances offers a chance of testing the AGB models at low metallicity and constrain the nucleosynthesis codes. We analyzed a total of $13 \mathrm{C}$-enhanced metal poor stars, of which 12 turned out to be $s$-process enriched. The combination of our results with the analyses already published in the literature allows to highlight the discrepancies present between the current state of the art AGB low metallicity models, suggesting that they are still lacking some ingredient.
\end{abstract}

Keywords. Stars: AGB and post-AGB, stars: chemically peculiar, binaries: general

\section{Introduction}

The largest so far metal poor stars searches (HK by Beers et al. 1992 and HES Christlieb et al. 2001) agree in finding that a considerable fraction of stars at low metallicity $([\mathrm{Fe} / \mathrm{H}]<-2.5 \mathrm{dex})$ is characterized by a considerable $\mathrm{C}$-overabundance $([\mathrm{C} / \mathrm{Fe}]>$ +1.0 dex). The subsequent high resolution studies of these $\mathrm{C}$-enhanced metal poor stars (CEMP) have uncovered a puzzling zoo of different peculiar chemical compositions: a few CEMP show overabundances of $r$-process elements (CEMP-r), some show no enhancement in the content of $n$-capture elements (CEMP-no), while the majority is characterized by anomalously high abundances of $s$-process elements (CEMP-s). While the origin of the abundance patterns of the two first classes is still not clear, observational data are consistent with CEMP-s being all members of double systems (Lucatello et al. 2005). Therefore, they likely owe their peculiar chemical pattern to the transfer of shell processed material from a more massive companion which has already evolved and undergone its AGB phase.

Hence the study of CEMP-s offers a chance to gain insight on AGB nucleosynthesis at low metallicity. So far most studies on CEMP-s concentrated exclusively or mostly on the heavy element, while the measurements of the abundances of some of the lights element (C, N, Na and $\mathrm{Li}$ ) as well as the $\mathrm{C}$ isotopic ratios are crucial to effectively constrain the models.

\section{Sample selection, data reduction and analysis}

With the aim of building up a sample of CEMP-s for which a uniform abundance analysis of a wide array of element is available, we observed 13 CEMP stars, 4 of which were already been analyzed in the literature, while 9 are newly discovered objects. The targets have been selected from the HK surveys and the HES survey on the basis of their low resolution metallicity $([\mathrm{Fe} / \mathrm{H}]<-2.5 \mathrm{dex})$, C-enhancement $([\mathrm{C} / \mathrm{Fe}]>+1.0 \mathrm{dex})$ and 


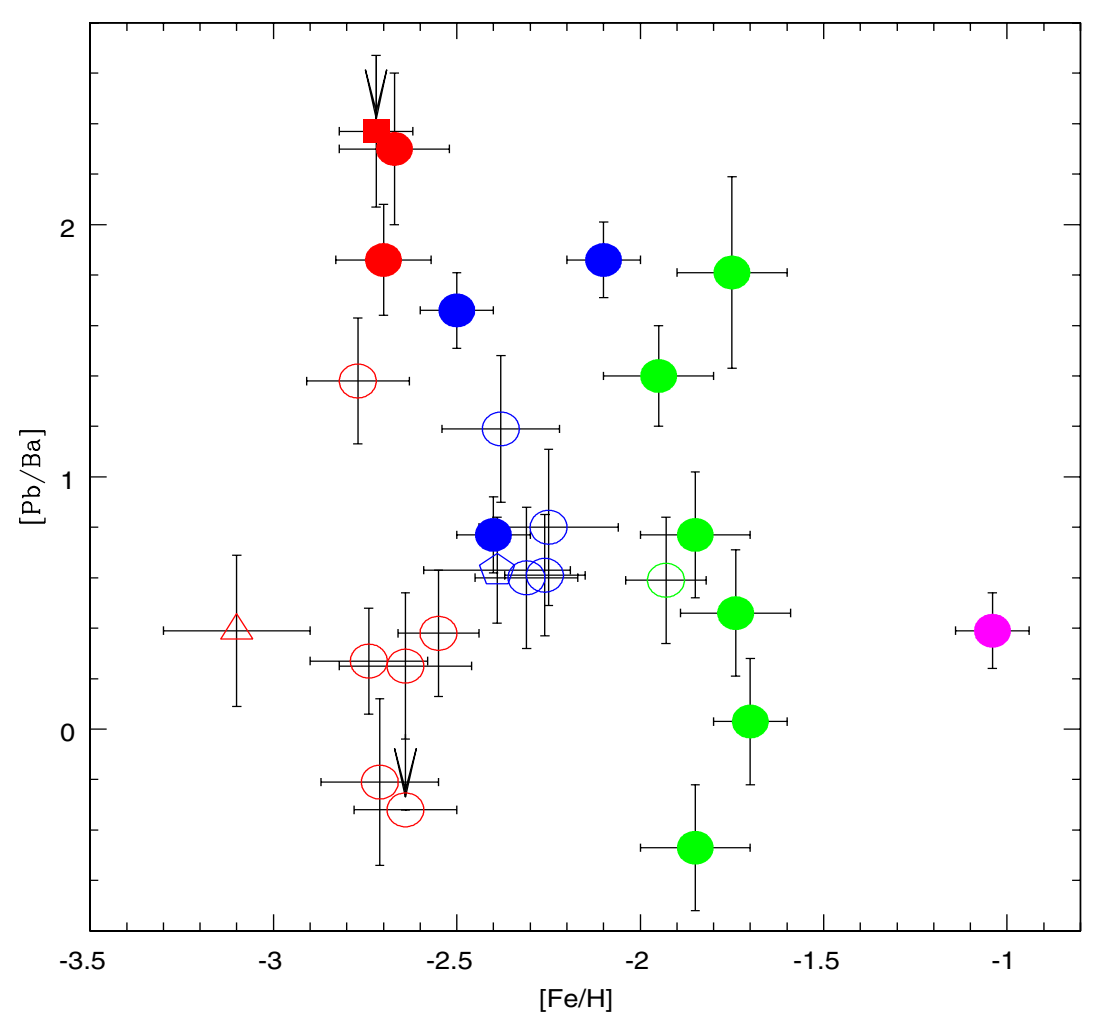

Figure 1. $[\mathrm{Pb} / \mathrm{Ba}]$ as a function of $[\mathrm{Fe} / \mathrm{H}]$ for the present sample plus literature stars. The colors code the metallicity, with red for $[\mathrm{Fe} / \mathrm{H}]<-2.5 \mathrm{dex}$, blue for $-2.5<[\mathrm{Fe} / \mathrm{H}]<-2.0$, green for $-2.0<[\mathrm{Fe} / \mathrm{H}]<-1.5$ and magenta for $[\mathrm{Fe} / \mathrm{H}]>-1.5$ dex. The shape of the symbols provides information about the abundance peculiarity of the objects. Open symbols are literature data, while closed ones are the present sample. The circles indicate CEMP-s, the squares CEMP-no and the triangle is CS 22892-52, a CEMP-r.

photometric temperatures $\left(\mathrm{T}_{\text {eff }}>4800 \mathrm{~K}\right)$. Seven objects, those from the HK survey, were observed with UVES at VLT (resolution $\mathrm{R} \simeq 50,000$ ) while the remaining 6 , from the HES, were observed with HIRES at Keck $(\mathrm{R} \simeq 45,000)$. The typical signal-to-noise ratio for the spectra is of about 100 per pixel). The spectral extraction and calibration was performed using IRAF.

Many other authors in the literature adopt a scale essentially based on $B-V$ for CEMP analysis. However, the presence of the $\mathrm{CH}$ G-band does affect the flux in the $B$ band, increasing the value of the $B-V$ color and thus yielding a too low temperature. To avoid this problem, we adopted a temperature scale based on the $V-K$ color, which is negligibly affected by the presence of $\mathrm{CH}, \mathrm{CN}$ and $C_{2}$ lines. The abundance analyses have been performed both by using equivalent widths (Fe, O, Na, Mg, Si, Ca, Sc, Ti, $\mathrm{Mn}, \mathrm{Sr}, \mathrm{Ba}, \mathrm{Ce}$ and $\mathrm{Nd}$ ) as well as spectral synthesis (C abundance and isotopic ratios, $\mathrm{N}, \mathrm{Eu}, \mathrm{La}$ and $\mathrm{Pb}$ ).

The comparison between our abundance measurements and that already available for the four objects for which analyses were already published in the literature show that our metallicities are consistently higher. This is easily explained by the above-mentioned choice of temperature scale, which, being higher than the literature analyses, leads to a 


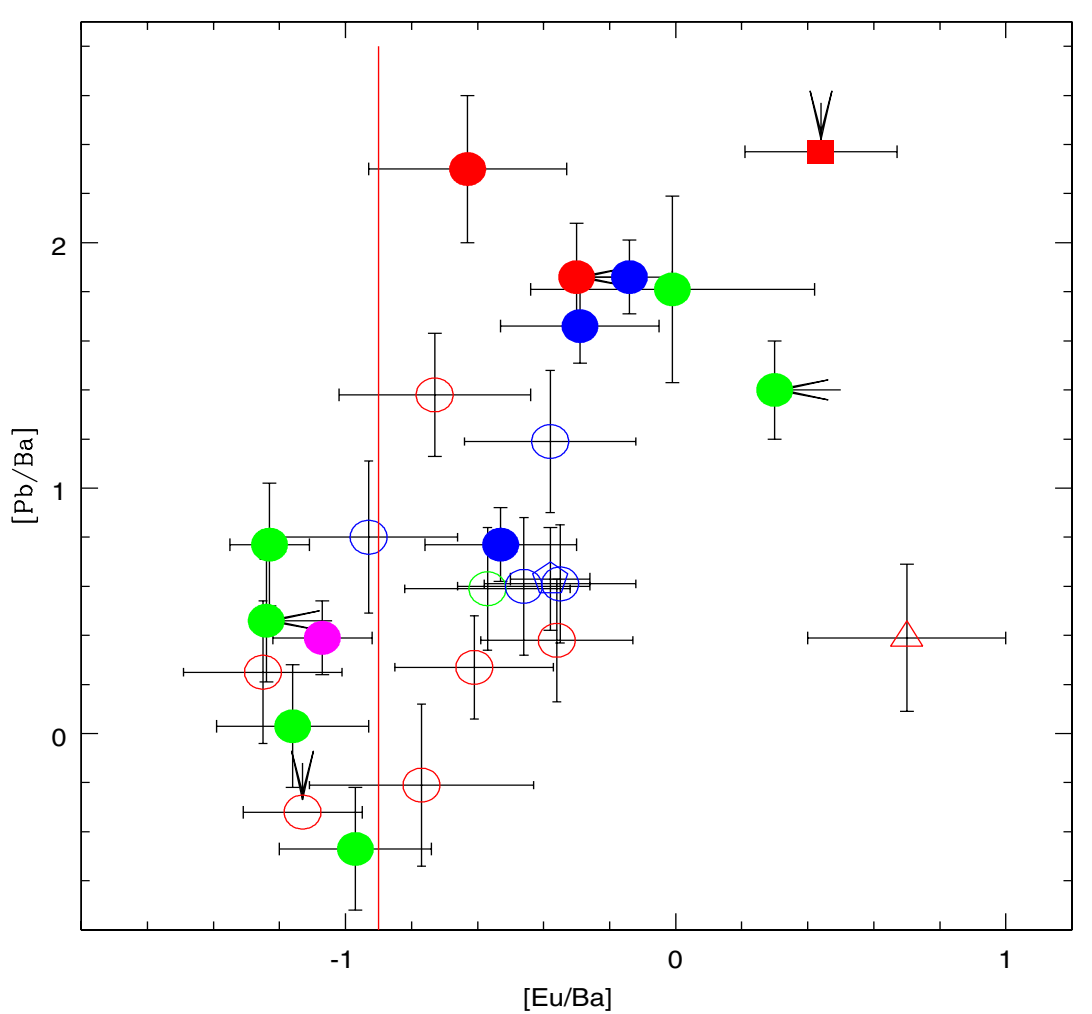

Figure 2. Same symbol coding as previous figure. This plot shows a correlation between $[\mathrm{Eu} / \mathrm{Ba}]$ and $[\mathrm{Pb} / \mathrm{Ba}]$.

higher value of the measured metal content. On the other hand, the abundance ratios relative to $\mathrm{Fe}$ of $\mathrm{C}, \mathrm{N}$ and $n$-capture elements for the four stars already analyzed, were found to be perfectly consistent with that given in the literature. Of 13 objects we analyzed, 12 turned out to be CEMP-s. We want to point out that there was no bias in our selection criteria in favor of $s$-process enhancement.

\section{The results}

The database built by combining the analyses published in the literature with our results provides interesting informations about the nucleosynthesis processes which originate the abundance anomalies. As it is shown in Fig. 1, there seems to be no dependence of the $s$-process efficiency (represented by $[\mathrm{Pb} / \mathrm{Ba}]$ ) with the metallicity. Although the models (see e.g. Busso et al. 2001) do expect that, as the number of available neutrons for seed nuclei increases (because of the decrease in metallicity), the s-process path shifts more toward the third peak increasing the $[\mathrm{Pb} / \mathrm{Ba}]$ ratio. This effect is not visible in the data, which require variable amount of ${ }^{13} \mathrm{C}$ in the pocket to explain the different values of $[\mathrm{Pb} / \mathrm{Ba}]$ at a given metallicity (Busso et al. 2001).

Keeping the ${ }^{13} \mathrm{C}$ pocket mass as a free parameter, the models are generally satisfactorily accounting for the observed abundance patterns, with some notable exceptions. In fact, the observed $\mathrm{Eu}$ abundances (as well as the $[\mathrm{Eu} / \mathrm{Ba}]$ or $[\mathrm{Eu} / \mathrm{La}]$ abundance ratios) are 


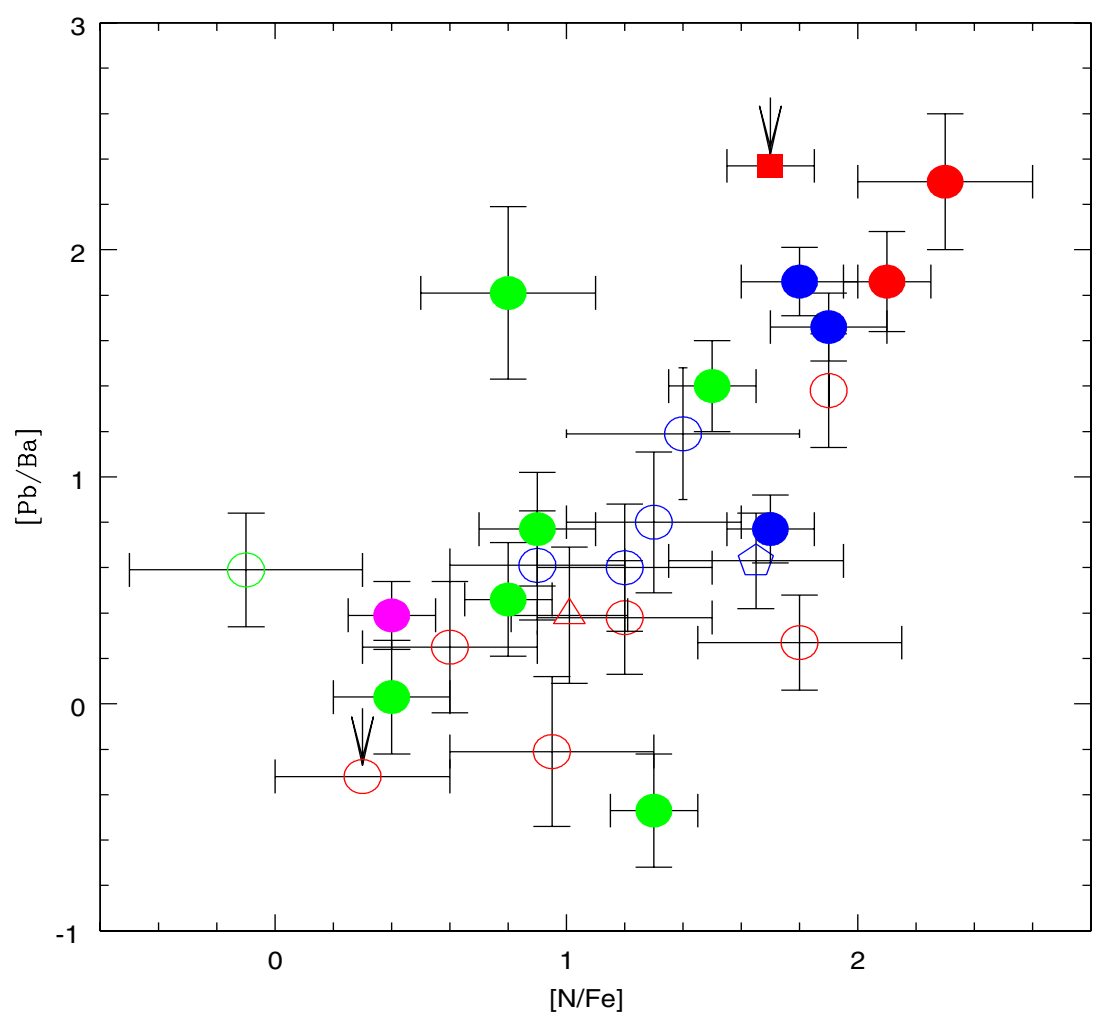

Figure 3. Same symbol coding as Figure 1. This plot compares the $[\mathrm{Pb} / \mathrm{Ba}]$ with $[\mathrm{N} / \mathrm{Fe}]$, showing a definite correlation, especially when considering the stars more metal poor than $[\mathrm{Fe} / \mathrm{H}]<-2.0$ dex. This result is somewhat surprising, as $\mathrm{N}$ is a poison for the $s$-process.

systematically higher than what predicted by the models (see Ivans et al. 2005 and references therein). Fig 2 shows the behavior of $[\mathrm{Eu} / \mathrm{Ba}]$ (which is traditionally adopted as an indicator of $r v s$. $s$-process origin of the heavy elements in a star) with respect to the $s$-process efficiency indicator, $[\mathrm{Pb} / \mathrm{Ba}]$. A considerable fraction of the CEMP-s stars studied so far is characterized by a $[\mathrm{Eu} / \mathrm{Ba}]$ of -0.5 dex or more, which is not easily explained in the current state of the art low metallicity AGB models. The data seem also to suggest that the CEMP-s which have the highest Eu overabundance are those with the the most efficient $s$-process (i.e. higher $[\mathrm{Pb} / \mathrm{Ba}]$ a values), which is again completely unaccounted for in AGB models.

Another interesting and unexpected result is the correlation present between the $\mathrm{N}$ abundance and the $s$-process efficiency (see Fig. 3). This is not accounted for in the AGB models at low metallicity which all predict $\mathrm{N}$ abundances (and $[\mathrm{N} / \mathrm{C}]$ ) much lower than the measured ones. A possibility to explain such a high $\mathrm{N}$ abundances could be that discussed by Herwig et al. (2004). They noted that for the handful of CEMP-s stars with a measured orbital period the latter anti-correlates with the measured $\mathrm{N}$ abundance, suggesting that the presence of a close companion could trigger mixing in the AGB star, enhancing the $\mathrm{N}$ production. However, in order to test this scenario, a much larger sample of CEMP-s with orbital solutions is needed. 
With this in mind, we have started a large collaboration aimed at monitoring the radial velocities of a large sample $(\sim 35)$ of CEMP-s. A fraction of the data has already been obtained using various telescopes (e.g. SARG at TNG, UES at WHT, FEROS at ESO $2.2 \mathrm{~m}$, UVES at VLT). Further observations will be performed in the upcoming months and more are planned in the next year. We will thus likely determine the orbital elements for a sample of CEMP-s large enough to make any inference based on their orbital characteristics statistically significant.

\section{Acknowledgements}

We would like to acknowledge N. Christlieb for selecting the HES targets and J. Cohen for providing the HIRES spectra used for this analysis.

\section{References}

Beers, T.C., Preston, G.W., \& Shectman, S.A. 1992, AJ, 103, 1987

Busso, M., Gallino, R., Lambert, D.L., Travaglio, C., \& Smith, V.V. 2001, ApJ, 557, 802

Christlieb, N., Green, P.J., Wisotzki, L., \& Reimers, D. 2001b, A\&A, 375, 366

Herwig, F. et al. in Chemical Abundances and Mixing in Stars in the Milky Way and its Satellites, Randich and Pasquini editors, 2004 Springer-Verlag Series "ESO Astrophysics Symposia" Ivans, I. et al., 2005, astro-ph/0505002

Lucatello, S., Tsangarides, S., Beers, T.C., Carretta, E., Gratton, R.G., \& Ryan, S.G. 2005, ApJ, 625, 825 


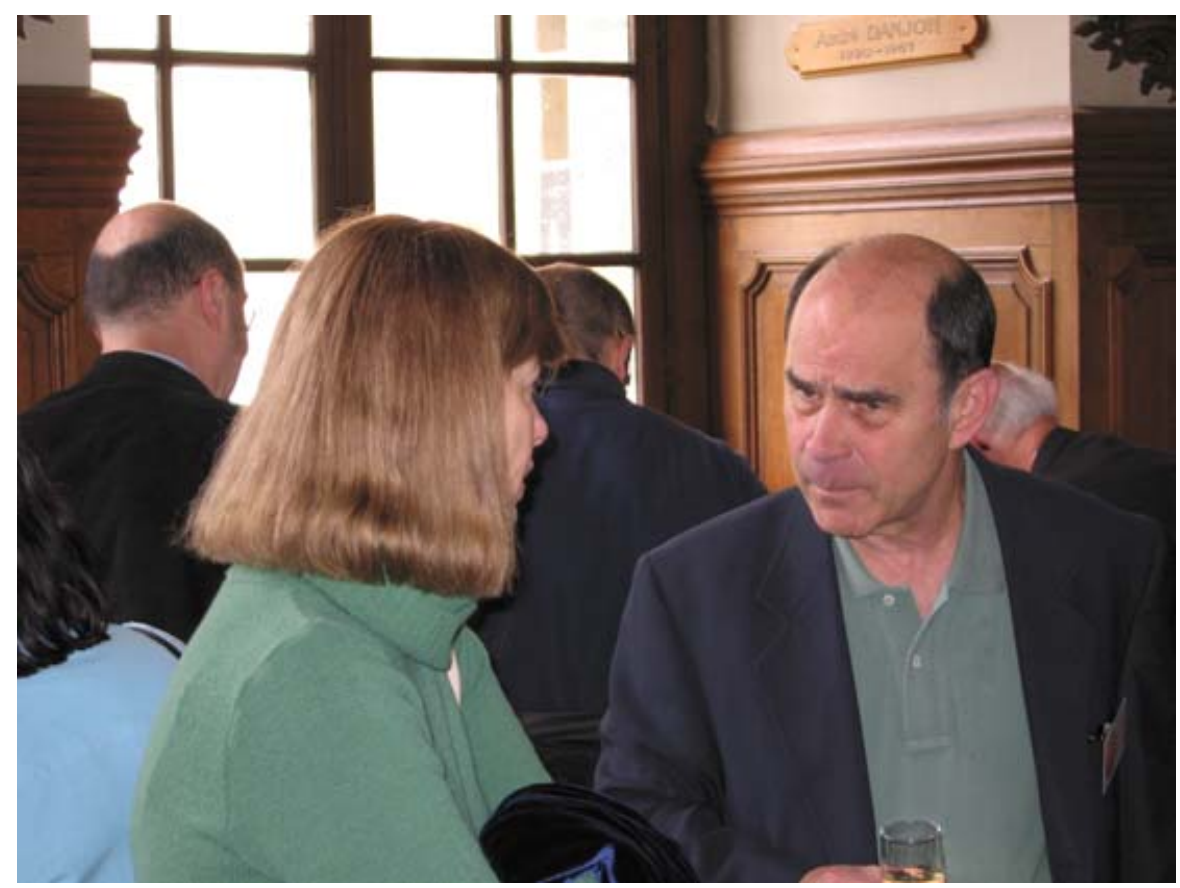

Jim Truran and Beatriz Barbuy at the welcome reception at the Paris Observatory.

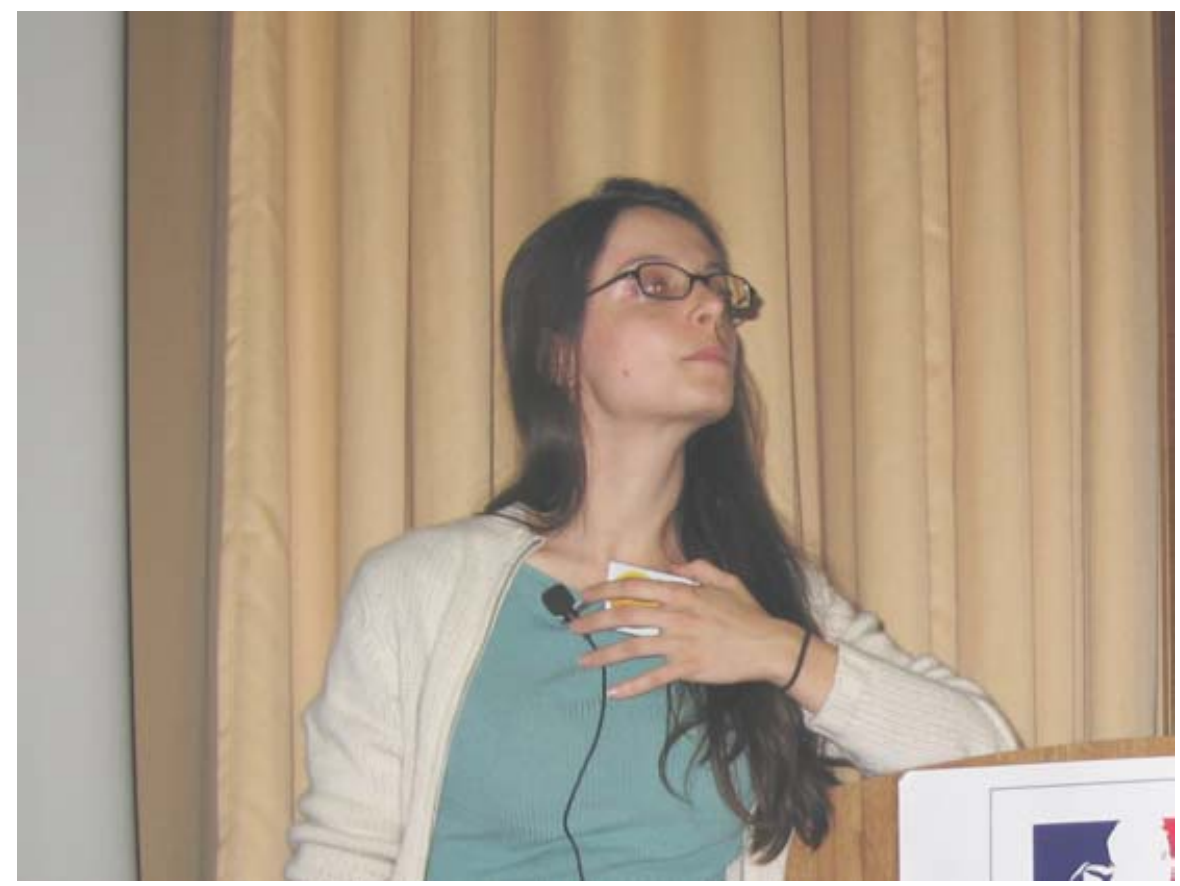

Sara Lucatello talking about s-process elements in carbon-enhanced stars. 